\title{
Characterization of new microsatellite markers based on the transcriptome sequencing of Clematis finetiana
}

\author{
Zhigao Liu' ${ }^{1,2}$, Weili Shao ${ }^{2}$, Yamei Shen², Mengcheng $\mathrm{Ji}^{2}$, Wenchao Chen², Ying Ye ${ }^{2}$ and Yongbao Shen ${ }^{\text {** }}$
}

\begin{abstract}
Background: Clematis is the biggest genus in the family Ranunculaceae with about 300 species. Clematis is also a globally important commercial group of flowers, especially in the United States and European countries. Their petals with different colors and shapes make the genus the "Queen of the Vines". However, the genomic information and phylogeny of Clematis based on existing molecular studies are limited. In this paper, new microsatellites (SSR) markers were identified from the transcriptome data of C. finetiana obtained using the Illumina paired-end sequencing technology.

Results: Sequences on a total of 71,900 high-quality unigenes with the mean length of 865 bp were produced in this study. There were 6192 unigenes annotated and classified into 49 functional sub-groups in three main ontology categories in GO (Gen Ontology) database,14,022 unigenes mapped to COGs (Clusters of Orthologous Groups) database and classified into 25 functional categories, and 21,494 unigenes obtained and divided into 128 pathways of KEGG (Kyoto Encyclopedia of Genes) Database. A total of 7532 SSRs were discovered from 6337 unigenes. We randomly tested 210 primer pairs, of which 52 primer pairs were able to generate specific products, and 19 possessed polymorphism in the 13 wild populations of six species from Clematis, which were used as a test material.
\end{abstract}

Conclusions: The dataset of C. finetiana transcriptome and the identified new SSR markers will promote genetic research and breeding effort in Clematis.

Keywords: Clematis finetiana, Marker development,Transcriptome sequencing, SSRs

\section{Background}

There are about 300 species [1] in the genus Clematis L., mainly distributed in the temperate zone of the northern hemisphere [2].China has abundant germplasm resources of Clematis, 147 species (49\%) were distributed throughout the country, especially in the southwestern area [3]. Clematis is the largest genus in the family Ranunculaceae, consists of typically vigorous, woody, climbing vines, and is famous for its diverse flower shapes and colors [4]; hundreds of cultivars make it the "Queen of the Vines". $C$. finetianais widely distributed in south China, in Zhejiang Fujian, Guangxi, Sichuan and Yunnan provinces. It is exploited and used as a medicinal plant because of

\footnotetext{
* Correspondence: 1692362952@qq.com

${ }^{1}$ College of Landscape Architecture, Nanjing Forestry University, Nanjing

210037, Jiangsu, People's Republic of China

Full list of author information is available at the end of the article
}

triterpenoid saponins, flavonoids and many other compounds present in roots and leaves [5]. C. finetiana is an evergreen species, which enhances its use in balconies and for fences. It can keep growing vigorously in hot summer conditions, which makes it different from many other cultivars and species of Clematis. C. finetiana is loved by home gardening enthusiasts for its lovely white flowers and excellent heat-resistance properties. It is a good resource for heat tolerance breeding of ornamental Clematis.

Microsatellite (SSR) markers are an important tool for the evaluation of genetic diversity and differentiation between species and populations [6, 7]. This marker type has a generally good transferability between closely related species and it is a useful tool in genetic mapping as well [8-10]. Previously, only few molecular marker studies and DNA sequencing-based investigations have 
been reported on Clematis, including the use of ISSR primers [11], randomly amplified polymorphic DNA [12], ITS sequencing [13, 14], and single nucleotide polymorphisms (SNP) analyses of chloroplast regions accD, rps16, rpl16, trnS-trnG,atpB-rbcL, trnV-atpE and matK $[15,16]$. Currently, there is a lack of SSR markers capable of effectively detecting polymorphisms in Clematis.

Transcriptome sequencing has been widely used for characterizing transcriptional events in a specific tissue or during a given period. It is a very useful tool also for research on non-model species that lack sequenced genome information. The high-throughput character and low cost makes RNA-seq a good choice for genetic investigations. The data resulting from transcriptome sequencing is valuable also for molecular marker development, such as microsatellite (SSR) and single nucleotide polymorphism (SNP) markers. To the best of our knowledge, very few research reports are available about the application of RNA-seqin studies on Clematis [17] and no information of SSR markers in Clematis has been published. To improve precision in genetic analyses on Clematis, we developed SSR markers based on the transcriptome sequencing of $C$. finetiana and utilized them to investigate inter- and intraspecific diversity and differentiation and genetic relationships among Clematis samples.

\section{Methods}

\section{Materials and methods Plant materials}

Young leaves, stems and roots of C. finetiana were collected from three individuals. All samples were frozen in liquid nitrogen immediately and stored at $-80{ }^{\circ} \mathrm{C}$ until RNA extraction. Young leaves of wild Clematis germplasm of six species including112 individuals (Additional file 1) were also harvested and dried with silica gel prior to DNA extraction.

\section{RNA extraction, CDNA library contraction and RNA-seq sequencing}

Total RNA was isolated using the TRIzol reagent (Invitrogen, Carlsbad, CA, USA) and RNeasy mini kit (Qiagen, Valencia, CA) according to the manufacturer's protocol. The quality and concentration of RNA were assessed by electrophoresis on a $1.2 \%$ agarose gel and using Nanophotometer Pearl/P360 (Implen, Munich, Germany). Equal amounts of purified RNA from different tissues were pooled together for cDNA library construction and transcriptome sequencing. TransCript cDNA sample prep kit (TransGen Biotech, China) was used for cDNA library construction. Agilent 2100 Bioanaylzer (Agilent Technologies, Palo Alto, CA, USA) and1.2\% agarose gel electrophoresis were used in qualification of the cDNA library. Then the library was sequenced using Illumina $\mathrm{HiSeq}^{\mathrm{sw}} 2000$ (Illumina).

\section{Data filtering, de novo assembly and unigene function annotation}

SeqPreq (https://github.com/jstjohn/SeqPrep)and sickle (https://github.com/najoshi/sickle) were used to remove sequencing adapters and trim low-quality sequences. After that, Trinity [18] software was used to assemble all clean high-quality reads. The expression level of transcripts was measured by RSEM, and the result was reported by units of TPM (transcripts per million).BLASTX was employed to annotate the function of unigene sequences using no redundant $(\mathrm{Nr})$ protein database $(\mathrm{NCBI})$, Swiss Prot database, KEGG (Kyoto Encyclopedia of Genes and Genomes), COG (Clusters of Orthologous Groups) and InterPro database with an $\mathrm{E}$-value $<10^{-5}$. Blast2GO software was used for gene ontology (GO) annotation.

\section{Real-time quantitative RT-PCR for verifying gene expression profiles}

The equivalent mixed RNA samples (young leaves, stems and roots) from three individuals of $C$. finetiana which used for transcriptome sequencing were used as three biological replicates for the qRT-PCR experiment. And 12 genes with different expression levels were randomly selected for validating the expression results of RNA-seq sequencing. Primers (Additional file 2) were designed by Primer Premier 5(http://downloads.fyxm.net/downloadnow-Primer-Premier-Others-Home-\&-Education-101178. html) based on the selected unigene sequences. PCR reaction mixture was consisted of $7 \mu \mathrm{l} \mathrm{dd}_{2} \mathrm{O}, 1 \mu \mathrm{l}$ cDNA, $10 \mu \mathrm{l} 2 \times \mathrm{SYBR}^{\circ}$ Select Master Mix (Applied Biosystems, VIC, Australia), and $1 \mu$ leach primer. qRT-PCR was carried out on ABI ViiA ${ }^{\text {ts }} 7$ Real-Time PCR system (Applied Biosystems, CA, USA) followed the cycling conditions: $95^{\circ} \mathrm{C}$ for $2 \mathrm{~min}, 50$ cycles at $95{ }^{\circ} \mathrm{C}$ for $10 \mathrm{~s}, 6{ }^{\circ} \mathrm{C}$ for $10 \mathrm{~s}$ and $72{ }^{\circ} \mathrm{C}$ for 40 s. GAPDH [19] was used as an internal control for normalizing the expression level of the 12 genes used for qRT-PCR testing. The specific primers of GAPDH were listed in (Additional file 2). The relative expression level of the selected gene was calculated via the $2^{-\Delta \mathrm{Ct}}$.

\section{SSR detection and primer design}

SSR discovery was performed using the MISA software (http://pgrc.ipk-gatersleben.de/misa) with the parameters (unit size-min repeats) as follows: $1-12,2-6,3-5,4-5$, 5-4, 6-4. Primer pairs of each detected SSR locus were designed by Primer3 (http://bioinfo.ut.ee/primer3) with default parameters. A total of 210 primer pairs (Additional file 3) were randomly chosen and synthesized for the validation of SSR markers.

\section{DNA extraction, validation of SSR markers and genetic analysis}

Total genomic DNA was extracted from dry leaf tissue of 112 wild individuals from 13 populations of six 
Clematis species (Additional file 1) using the E.Z.N.A Plant DNA Mini Kit Spin Protocol (Omega Bio-tek, GA, USA) according to manufacturer's instructions. The quality and concentration of DNA were determined by Nanophotometer Pearl/P360 (Implen, Munich, Germany).

\section{PCR amplification and data analysis}

PCR reactions for SSR regions were carried out in $20-\mu \mathrm{l}$ volumes by mixing the following components: $11 \mu \mathrm{l}$ of $\mathrm{dd}_{2} \mathrm{O}, 2 \mu \mathrm{l}$ of $10 \times$ buffer, $0.4 \mu \mathrm{l}$ of $10 \mathrm{mM}$ dNTP, $0.6 \mu \mathrm{l}$ of Dynazyme II DNA polymerase (Thermo Fisher Scientific, $2 \mathrm{U} / \mu \mathrm{l}$ ), $2 \mu \mathrm{l}$ of genomic DNA (about $20 \mathrm{ng}$ ) and $2 \mu \mathrm{l}(5 \mathrm{pmol} / \mu \mathrm{l})$ of both primers. The PCR reactions were carried out with an initial denaturation at $94{ }^{\circ} \mathrm{C}$ for $45 \mathrm{~s}$, followed by 35 cycles of $30 \mathrm{~s}$ at $94{ }^{\circ} \mathrm{C}, 30 \mathrm{~s}$ at SSRspecific annealing temperature (Table S2) and $40 \mathrm{~s}$ of elongation at $72{ }^{\circ} \mathrm{C}$, and with a final elongation at $72{ }^{\circ} \mathrm{C}$ for $5 \mathrm{~min}$. After amplification, the PCR products were diluted at 1:2-1:5 (depending on the concentration) with Milli-Q water. DNA fragments were analyzed using a capillary electrophoresis system Qsep 100DNA Analyzer (BiOptic, Taiwan, China) [20]. The observed numbers of alleles $(\mathrm{Na})$, effective numbers of alleles $(\mathrm{Ne})$, expected heterozygosities $(\mathrm{He})$ and observed heterozygosities (Ho) were determined by Popgene1.32 [21]. Polymorphism information contents (PIC) were estimated by PowerMarker V3.25 [22] (Liu and Muse, 2005). Phylogenetic trees were constructed by PowerMarker V3.25 using the UPGMA method based on Nei's (1979) genetic distances [23].

\section{Results}

\section{RNA-seq sequencing and de novo assembly}

A total of $11.12 \mathrm{~Gb}$ clean bases were produced from the transcriptome sequencing of C. finetiana. $111.18 \mathrm{Mb}$ (99.91\%) clean reads with $97.56 \%$ Q20 and 93.66\% Q30 were collected through data filtering. 71,900 unigenes were generated when all high-quality reads were assembled. The total length, mean length, N50 and GC content of these unigenes were 62,250, $865 \mathrm{bp}, 1469 \mathrm{bp}$, and $42.35 \%$ (Table 1). The lengths of 21,676 (30.15\%) unigenes were between 300 and $400 \mathrm{bp}, 43,200$ (60.08\%) unigenes were between 400 and 2000 bp, 4760 (6.62\%) unigenes were between 2000 and 3000, and 2264 (3.15\%) unigenes were longer than 3000 bp (Fig. 1).

\section{Functional annotation}

38,814 (53.98\%) out of 71,900 unigenes were annotated by at least one of the following databases: Nr, Swissprot, KEGG, COG, Interpro and GO. Nr database was the largest matched database with 36,015 unigenes $(50.09 \%$ of all unigenes) annotated, followed by the Swissprot $(23,982,33.35 \%)$, KEGG $(21,494,29.89 \%)$, COG (1022, 19.50\%), and Interpro (27,004, 37.56\%) database (Table 1). The $\mathrm{Nr}$ annotation indicated that $58 \%$ of
Table 1 Summary of transcriptome data for C. finetiana

\begin{tabular}{ll}
\hline Item & Number \\
\hline 1. Raw sequences and assembly statistics & \\
Total amount of clean reads(Mb) & 111.18 \\
Total amount of clean bases(Gb) & 11.12 \\
GC content percentage (\%) & 42.35 \\
Clean reads proportion (\%) & 99.91 \\
Total number of unigenes, & 71,900 \\
Mean length of unigenes(bp), N50(bp), GC content(\%) & $865,1469,42.35$ \\
2.Statistics of unigene annotation & \\
Gene annotation against Nr (\%) & $36,015(50.09 \%)$ \\
Gene annotation against Swiss-Prot (\%) & $23,982(33.35 \%)$ \\
Gene annotation against KEGG (\%) & $21,494(29.89 \%)$ \\
Gene annotation against COG (\%) & $14,022(19.50 \%)$ \\
Gene annotation against GO (\%) & $6192(8.61 \%)$ \\
Gene annotation against Interpro (\%) & $27,004(37.56 \%)$ \\
All annotated genes (\%) & $38,814(53.98 \%)$ \\
\hline
\end{tabular}

unigenes showed high homology with e-values under $1 \mathrm{e}-30,41 \%$ of unigenes showed very high homology with e-values under 1e-60 (Fig. 2a). The top-hit species in similarity search against the $\mathrm{Nr}$ database included Nelumbonucifera (14,304, 39.72\%), Vitisvinifera (4652, 12.92\%), Theobroma cacao (1196, 3.32\%), Citrus sinensis (929, 2.58\%) and others (14,923, 41.47\%) (Fig. 2b).

The GO database was employed to predict the possible function of $C$. finetiana unigenes. 6192 unigenes were annotated and classified into 49 functional sub-groups in three ontology categories, including biological processes, molecular functions and cellular components (Fig. 3a). Biological processes was the largest category with 12,526 unigenes, followed by cellular components (9289 unigenes) and molecular functions (7381 unigenes). The main groups within biological processes included metabolic processes $(3495,56.44 \%)$, cellular processes $(2959,47.79 \%)$ and single-organism processes (2230, 36.01\%). Cell (2101, 33.93\%), cell parts $(2101,33.93 \%)$ and organelles $(1496,24.16 \%)$ were the most frequent groups in the cellular components category. Catalytic activity (3596, 58.08\%), binding (2840, 45.87\%) and transporter activity $(371,5.99 \%)$ were the most frequent groups in the molecular functions category. According to the COG database, a total of 14,022unigenes were classified into 25 functional categories, which covered most life processes (Fig. 3b). General function was the biggest category with 4045 unigenes (6.85\%), followed by the replication, recombination and repair category $(2144,3.63 \%)$ and the transcription category (2024, 3.43\%).

Pathway annotations were employed to further evaluate the biological functions of the unigenes. In all, 


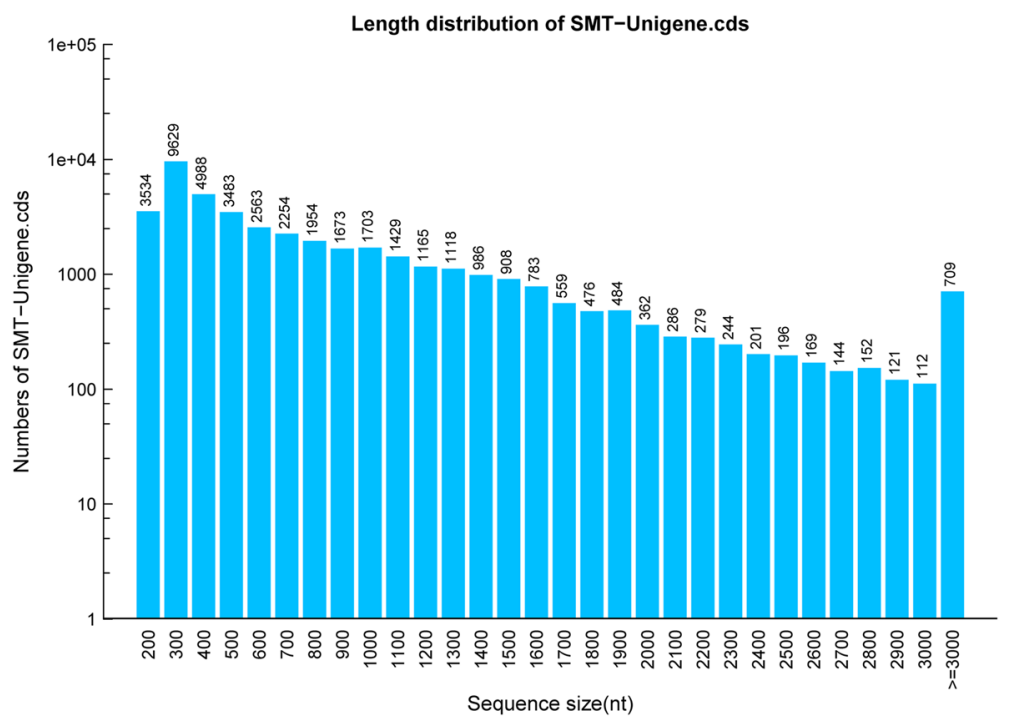

Fig. 1 Length distribution of all unigenes obtained from the C. finetiana transcriptome. The $x$-axis indicates a different sequence size, and the $y$-axis indicates the unigene numbers of a specific sequence size

21,494 unigenes were obtained and divided into 128 KEGG pathways (Additional file 4) which belonged to five level-1 categories, namely, cellular processes (1013, 4.71\%), environmental information processing (1430, 6.65\%), genetic information processing (6492, $30.20 \%)$, metabolism $(13,536,62.98)$ and organismal systems (1928, 8.67\%). In level-2 categories, global map (5275, 24.54\%), translation (2766, 12.87\%), folding, sorting and degradation (1902, 8.85\%), environmental adaptation (1873, 8.71\%) and carbohydrate metabolism (1611, 7.50\%) were the five largest categories.

\section{RT-qPCR validation of gene expression profiles}

In order to verify the reliability of the transcriptome sequencing result, 12 unigenes were randomly selected and the expression levels of them were evaluated via RT-qPCR (Fig. 4). Genes with similar expression levels in RNA-Seq (such as CL6568.Contig2, CL5267.Contig1 and CL1188.Contig1) showed a little undulating expression change in the results of RT-qPCR due to the different of sensitivity and algorithms. All tested genes presented similar change trends in RNA-Seq and qPCR experiment, indicating that the results of transcriptome sequencing were reliable.

\section{a}

$$
\begin{array}{ll}
\square & \square 0 \sim 1 \mathrm{e}-100 \\
\square 1 \mathrm{e}-100 \sim 1 \mathrm{e}-60 & \square 1 \mathrm{e}-60 \sim 1 \mathrm{e}-45 \\
\square 1 \mathrm{e}-45 \sim 1 \mathrm{e}-30 & \square 1 \mathrm{e}-30 \sim 1 \mathrm{e}-15
\end{array}
$$$$
1 \mathrm{e}-15 \sim 1 \mathrm{e}-5
$$

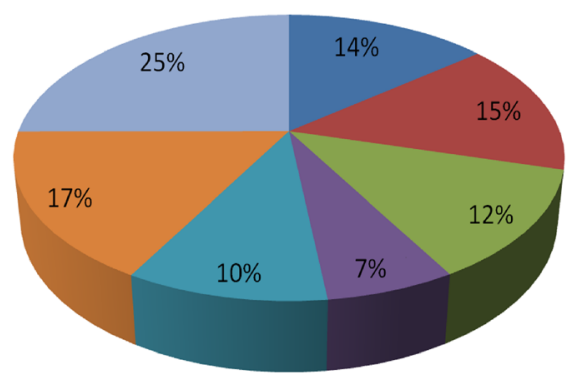

b

$$
\begin{aligned}
& \text { Species } \\
& \text { Nelumbo nucifera } \\
& \text { Vitis vinifera } \\
& \text { Theobroma cacao } \\
& \text { Citrus sinensis } \\
& \text { other }
\end{aligned}
$$

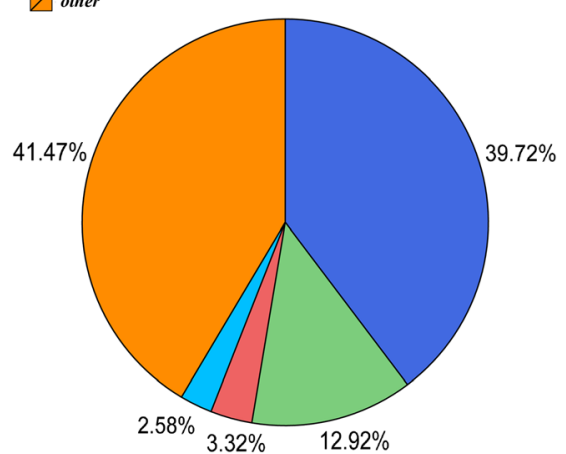

Fig. 2 Characteristics of homology search in C. finetiana unigenes. (a) E-value distribution of the BLASTx hits against the nr database. $\mathbf{b}$ Top-hit species in similarity search of unigenes 


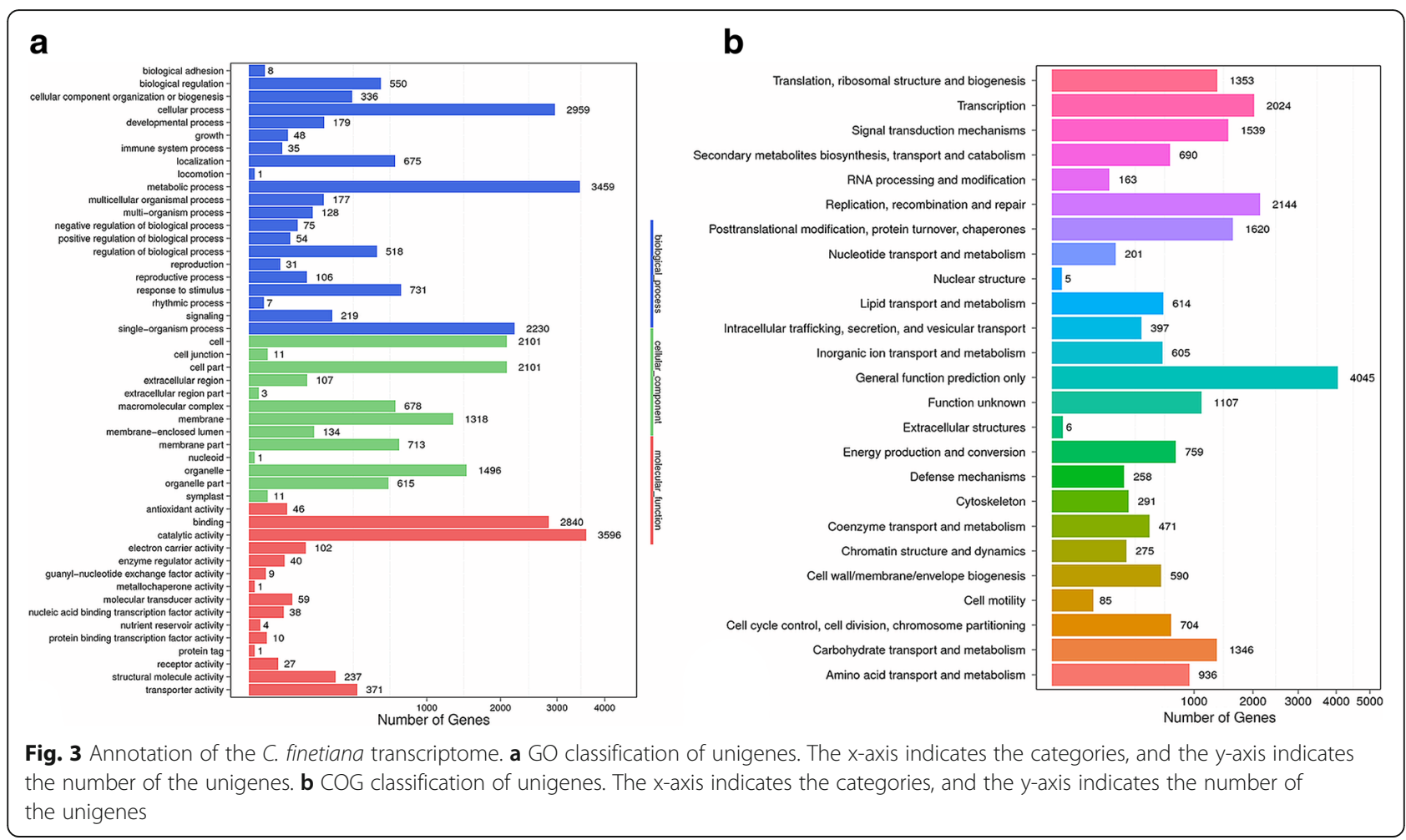

\section{Development and validation of SSR markers}

In this study, MISA perl script (http://pgrc.ipk-gatersleben.de/misa) was used to detect microsatellites in unigene sequences. A total of 7532 SSRs were recognized from 6337 unigenes (Table 2). Di-nucleotides were the most abundant type $(3114,41.34 \%)$, followed by trinucleotides $(2594,34.44 \%)$ hexa-nucleotides $(515,6.84 \%)$, penta-nucleotides $(126,1.67 \%)$ and tetra-nucleotides $(94$, $1.25 \%)$. AG/CT (2410, 37.40\%) was the most abundant motif type, followed by AAG/CTT (730, 11.33\%),
AC/GT (453, 7.03\%), ACC/GGT (387, 6.01\%), AAC/ GTT $(348,5.04 \%)$, ATC/ATG $(320,4.97 \%)$ and others (Fig. 5). The number of repeat motifs of SSR loci (except mono-nucleotides) ranged from 4 to 28 , SSRs with six motif repeats were the most common type (1715, 26.62\%), followed by five $(1537,23.68 \%)$, seven (947, 14.70\%) and eight repeats $(659,10.23 \%)$ types, respectively (Additional file 5).

In all, 210 SSR sites (Additional file 3) were randomly selected for primer designing. Among them, 52 (24.76\%)

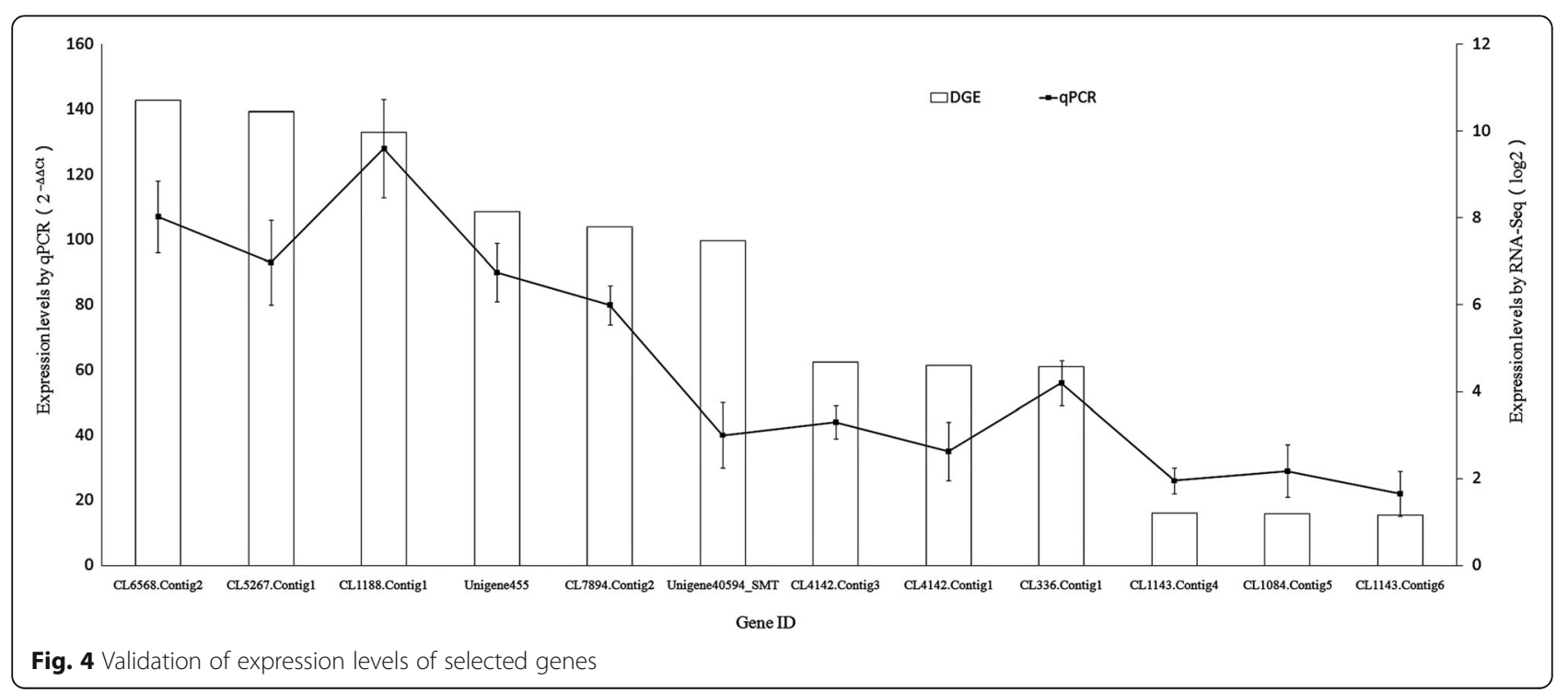


Table 2 Summary of microsatellite data of C. finetiana transcriptome

\begin{tabular}{ll}
\hline Item & Number \\
\hline Total number of sequences examined & 71,900 \\
Total size of examined sequences (bp) & $62,250,256$ \\
Total number of identified SSRs & 7532 \\
Number of SSR containing sequences & 6337 \\
Number of sequences containing more than 1 SSR & 961 \\
Number of SSRs present in compound formation & a \\
\hline aThe SSR locus containing at least 2 repeat motifs & 568
\end{tabular}

primer pairs generated expected specific products, 38 (18.10\%) primer pairs amplified PCR products much smaller or larger than the expect size, while the other 120 (57.14\%) primer pairs did not amplify at all. The other five species (C. brevicaudata, C. apiifolia, C. uncinata, C. lasiandra and C. henryi) were assessed to evaluate the transferability of the 52 SSR well working markers. The results showed that 19 loci were polymorphic, and a total of 135 alleles were discovered at those 19 SSR loci among the samples including 112 accessions from 13 populations of 6 Clematis species. The allele sizes were around the predicted allele sizes (Additional file 6; Additional file 7). The allele numbers per locus $(\mathrm{Na})$ rangedfrom 3 to 11 (average 7.11), the effective number of alleles (Ne) ranged from 1.80 to 9.65 (average 4.44). The ranges of expected homozygosity (Ho) and expected heterozygosity $(\mathrm{He})$ were from 0.10 to 0.57 (average 0.25 ) and 0.43 to 0.86 (average 0.75 ), respectively. The polymorphism information contents (PIC) per locus ranged from 0.41 to 0.88 (average 0.72), for the whole dataset (Additional file 8).

The UPGMA tree (Fig. 6) was constructed based on Nei's genetic distances. Two main clusters were generated. Different populations from C. apiifolia, C.finetiana and C. uncinata were grouped together, respectively. $C$. lasiandra and $C$. henryi belonged to group II and the other 4 species were distributed into another bigger group I, in which C. brevicaudata and C. apiifolia were

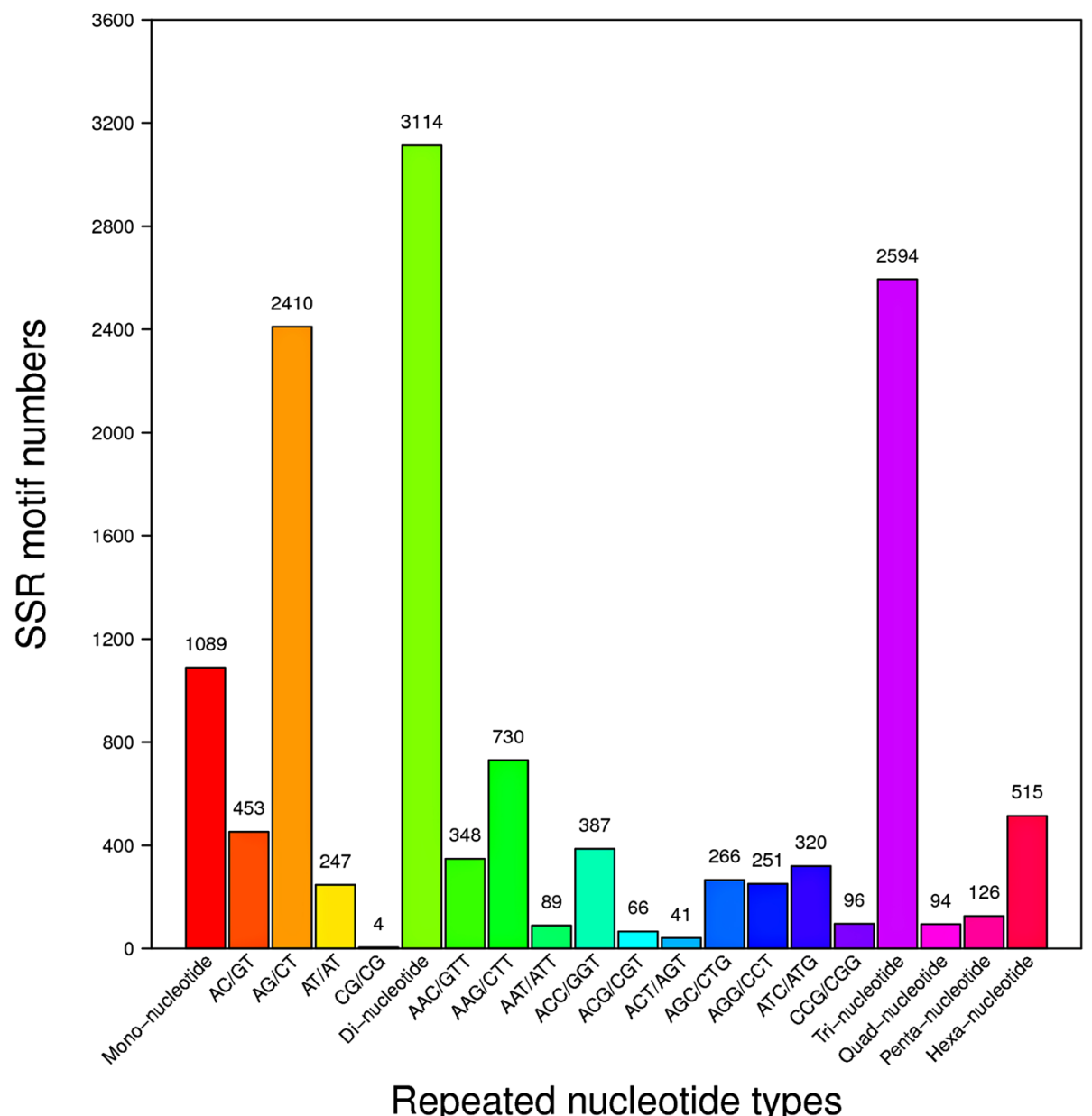

Fig. 5 Frequency distribution of SSR repeats types. Motif types of di-nucleotides and tri-nucleotides are represented. The x-axis indicates the categories, and the $y$-axis indicates the number of the unigenes 


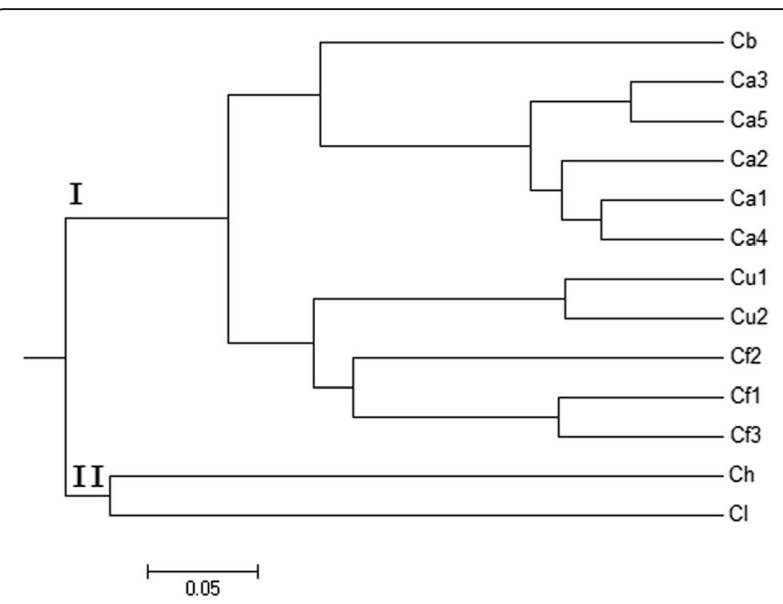

Fig. 6 Phylogenetic tree of 13 populations from 6 Clematis species based on 19 SSR loci and utilizing Nei's genetic distances. The population abbreviations are the same as those in Additional file 1

closely related, and C. uncinata and C.finetiana were grouped together.

\section{Discussion}

Clematis cultivars are well known for their diverse flower types and colors; especially the large flowered hybrids make Clematis one of the most popular flowers. However, only few molecular marker-based approaches have been used to assist breeding of ornamental Clematis. Inter-simple sequence repeat markers (ISSR) have been used to fingerprint 32 vining cultivars and five novining species for assessing genetic relationships and cultivar identification [11]. Random amplified polymorphic DNA (RAPD) has been used to confirm the identity of Clematis hybrids [12]. There are several earlier reports about the systematic classification and phylogeny research on Clematis, which are mainly based on phenotypic characteristics, such as the shape and length of sepals, the indumentum of filaments, the structure of pollen and plant morphology [2, 3, 24, 25]. A few reports have been published about the interspecific relationships among Clematis based on molecular markers [11, 14, 26, 27], but such research is still limited due to the lack of genomic information of Clematis. In this study, the transcriptome of C. finetiana was sequenced in order to obtain genomic data and to develop SSR markers.

In the present study, a total of 71,900 unigenes was obtained via transcriptome sequencing with an average length of $865 \mathrm{bp}$. The average length of sequenced $C$. finetiana unigenes was longer compared to recently released transcriptome sequencing results on Eucommia ulmoides (645 bp) [28], Vigna mungo (443 bp) [29] and Prunus sibirica (652 bp) [30]. As a result of BLAST searches, a total of 38,814 unigenes $(53.98 \%)$ were annotated by Nr, Swiss-Prot, COG, GO, Interpro and KEGG databases, while about 33,086 unigenes $(46.02 \%)$ were not annotated by any of these six databases, thus indicating that some unigenes may be particular to Clematis. Transcriptome sequencing is also useful tool for the development of SSR markers, and there are previous reports about the development of SSRs in plants through transcriptome sequencing [31-34].

Microsatelite markers are co-dominant, highly polymorphic and easily reproducible [35]. They are important tools to examine genetic diversity, the assessment of genetic relationships and population genetic structure in plants [26]. The application of SSR markers in the study of Clematis has been limited due to the expenses and time-consuming work when developing markers by traditional methods. Some other molecular marker systems have been applied to studies on Clematis. ITS sequences have been analyzed to provide molecular evidence for the identification of 14 medicinal Clematis species [14, 26]. The sequences of chloroplast DNA (atpB-rbcL spacer region, matK, trnK, trnL intron, andtrnL-trnF spacer region) and the nuclear actin I intron have been used for the analysis of phylogenetic relationships within the genus Clematis. The result showed that the taxonomic status of several species was not consistent with previous interspecific classifications based on morphology [15, 36]. However, none of them have utilized SSR markers.

In this study, a high quantity of high-quality transcriptome sequences was obtained, which could be used to develop SSR markers for Clematis. A total of 7532 SSRs were recognized from 6337 unigenes. These SSRs could be divided into five categories based on the number of bases from two to six, di-nucleotides and tri-nucleotides being the most abundant types, which is in concord with previous studies [30, 37]. Furthermore, AG/CT and AAG/CTT were the most abundant motif typesamongnucleotides and tri-nucleotides, similarly as previously reported [38]. Tetr-nucleotide and penta-nucleotide motifs of SSRs are usually less polymorphism in coding sequences region. Nevertheless, the two types were generally included in the list of screening primers [29-31]. In the present study, SSR primers of Clematis were screened for the first time. In consideration of the comprehensiveness of the experiment, a total of 210 primer pairs from all five SSR types (including tetr-nucleotide and penta- nucleotide motifs) were randomly chosen. We successfully developed 52 SSR markers, which gave clear amplification products and showed good transferability among different species of Clematis. Within the genus, 19 markers were polymorphic, and all except one of these polymorphic loci showed the presence of a high level of polymorphism with PIC values above 0.60. Several studies have indicated that tetra-nucleotide SSRs have high polymorphism rates [39]. In the present study, 
both two tetra-nucleotide SSRs showed high polymorphism, of then'4-11'was the most informative tetranucleotide SSR with the PIC value of 0.88 . Hexanucleotides $(9,56.25 \%)$ were the most abundant type among the 19 polymorphic SSRs, followed by trinucleotides $(4,21.05 \%)$ and di-nucleotides (3, 15.79\%). This observation was similar to the result of SSR development in the rubber tree [40]. All amplified fragments resulting from the 19 primer pairs were around the expected size and showing that the detected polymorphism resulted from the variation in the number of SSR repeats.

Microsatellite markers have been proved to be effective tool in the evaluation of interspecific genetic diversity and phylogenetic relationship. The phylogenetic tree constructed based on SSR data showed that $C$. brevicaudata and $C$. apiifolia belong to the same group; $C$. uncinata and C.finetiana are closely related. These results are similar to those of Xie et al., who used nuclear ITS and three plastid regions in the phylogentic analyses of Clematis [13]. This result demonstrated the effectiveness in analyzing Clematis genetic relationships and confirming the potential value of the transcriptome database for the development of new SSR markers. The newly developed polymorphic SSR markers can be applied into population genetic and phylogenetic studies, species and hybrid identification, and possibly also to marker-assisted breeding in Clematis.

\section{Conclusion}

The present study reported the functional characterization of transcriptome sequences of Clematis and development of new SSR markers, which are applicable to many kinds of studies in Clematis. To the best of our knowledge, this is the first report on the development and use of any kind microsatellite markers in the genus Clematis. They can be used in further studies on the genetic diversity, population genetics and phylogeography of Clematis, and they can assist the breeding of new ornamental cultivars.

\section{Additional files}

Additional file 1: Sampling information of the six Clematis species. (XLSX $10 \mathrm{~kb}$ )

Additional file 2: Used primers for qRT-PCR experiments. (XLSX $12 \mathrm{~kb}$ )

Additional file 3: Details of 210 selected SSR markers used for polymorphism validation. (XLSX $34 \mathrm{~kb}$ )

Additional file 4: The KEGG pathway annotations. (XLSX $10 \mathrm{~kb}$ )

Additional file 5: The frequency of classified SSR repeat types. (XLSX $24 \mathrm{~kb}$ )

Additional file 6: Detected allele sizes of 19 SSR loci in different Clematis species. (XLSX $12 \mathrm{~kb}$ )

Additional file 7: The product sizes of the amplified locus ' $6-73$ ' in 4 Clematis samples determined by Qsep 100 DNA Analyzer. (PNG 136 kb)

Additional file 8: Characteristics of 19 polymorphic SSR loci. (XLSX $11 \mathrm{~kb}$ )

\section{Abbreviations}

COG: Clusters of Orthologous Groups; GO: Gene Ontology; KEGG: Kyoto Encyclopedia of Genes and Genomes; Nr: Non-redundant; SSR: Simple sequence repeat marker

\section{Acknowledgements}

We would like to thank Fuqiang Cui, Helena Korpelainen, Chao Zhang, Bin Dong and Zaikang Tong for their suggestions and commenting on the paper.

\section{Funding}

This work was supported by Projects for floriculture special breeding of the Zhejiang province (2012C12909-4) and Major science projects for agriculture (floriculture) new variety breeding of the Zhejiang province (2016C02056-13-4).

Availability of data and materials

The datasets used and analyzed during the current study are available from the corresponding author on reasonable request.

\section{Authors' contributions}

$\mathrm{L}-\mathrm{ZG}, \mathrm{S}-\mathrm{YB}$ and J-MC developed the project and designed the experiments. $Y-Y$ and $C-W C$ performed the experiments. L-ZG, S-WL and S-YM wrote and revised the manuscript. All authors discussed the results and commented on the manuscript. All authors read and approved the final manuscript.

Ethics approval and consent to participate

Not applicable.

\section{Competing interests}

The authors declare that they have no competing interests.

\section{Publisher's Note}

Springer Nature remains neutral with regard to jurisdictional claims in published maps and institutional affiliations.

\section{Author details}

${ }^{1}$ College of Landscape Architecture, Nanjing Forestry University, Nanjing 210037, Jiangsu, People's Republic of China. ${ }^{2}$ College of Landscape Architecture, Zhejiang A \& F University, Hangzhou 311300 Zhejiang, People's Republic of China.

Received: 18 December 2017 Accepted: 2 May 2018

Published online: 15 May 2018

\section{References}

1. Wang WT, Bartholomew B. Clematis. In: Wu Z-Y, Raven P, editors. Flora of China, vol. 6. Beijing: Science Press, St. Louis; 2001. p. 97-165.

2. Hao DC, Gu XJ, Xiao PG, Peng Y. Chemical and biological research of Clematis medicinal resources. Chin Sci Bull. 2013;58(10):1120-9.

3. Wang WT, Li LQ. A new system of classification of the genus Clematis (Ranunculaceae). Acta Phytotaxon Sin. 2005:43(5):431-88.

4. Sheng L, Ji K, Yu L. Karyotype analysis on 11 species of the genus Clematis. Braz J Bot. 2014;37(4):601-8.

5. He YX, Li L, Zhang K, Liu ZR. Cytotoxic triterpene saponins from Clematis mandshurica. J Asian Nat Prod Res. 2011;13(12):1104-9.

6. Pedroni MJ, Sondgeroth KS, Gallegolopez GM, Echaide I, Lau AO. Comparative transcriptome analysis of geographically distinct virulent and attenuated Babesia bovis strains reveals similar gene expression changes through attenuation. BMC Genomics. 2013;4(1):763.

7. Rai HS, Mock KE, Richardson BA, Cronn RC, Hayden KJ, Wright JW, Knaus BJ, Wolf PG. Transcriptome characterization and detection of gene expression differences in aspen (Populus tremuloides ). Tree Genet Genome. 2013;9(4): 1031-41.

8. Yoo MJ, Wendel JF. Comparative evolutionary and developmental dynamics of the cotton (Gossypium hirsutum ) fiber transcriptome. PLoS Genet. 2014; 10(1):e1004073.

9. Ma H, Jiang W, Liu P, Feng N, Ma Q, Ma C, Li S, et al. Identification of transcriptome-derived microsatellite markers and their association with the growth performance of the mud crab (Scylla paramamosain). PLoS One. 2014;9(2):e89134. 
10. Varshney RK, Sigmund R, Börner A, Korzun V, Stein N, Sorrells ME, Langridge $P$, Graner A. Interspecific transferability and comparative mapping of barley EST-SSR markers in wheat, rye and rice. Plant Sci. 2005;168(1):195-202.

11. Gardner N, Hokanson SC. Intersimple sequence repeat fingerprinting and genetic variation in a collection of Clematis cultivars and commercial germplasm. J Am Soc Hortic Sci. 2005:40(7):1982-7.

12. Tao Y, Wang LY, Roh MS. Confirmation of Clematis hybrids using molecular markers. Sci Hortic-Amsterdam. 2010;125(2):136-45.

13. Xie L, Wen J, Li LQ. Phylogenetic analyses of Clematis (Ranunculaceae) based on sequences of nuclear ribosomal ITS and three plastid regions. Syst Bot. 2011;36(4):907-21.

14. Xiao LI, Wang P, Zhao L. rDNA-ITS sequence analysis of the traditional mongolian medicinal plants in Clematis L. J Inner Mongolia Medical College. 2012;34(4):325-30.

15. Slomba JM, Garey JR, Essig FB. The actin I intron-a phylogenetically informative DNA region in Clematis (Ranunculaceae). Sida Contributions to Botany. 2004;21(2):879-86.

16. Yan SX, Liu HJ, Lin LL, Liao S, Jin YL, Pei LY, Xie L. Taxonomic status of Clematis acerifolia var. elobata, based on molecular evidence. Phytotaxa. 2016;268(3):209

17. Gao L, Ma Y, Wang P, Wang S, Yang R, Wang Q, Li L, et al. Transcriptome profiling of Clematis apiifolia: insights into heat-stress responses. DNA Cell Biol. 2017:36(11):938-46.

18. Grabherr MG, Haas BJ, Yassour M, Levin JZ, Thompson DA, Amit I, Adiconis $X$, et al. Full-length transcriptome assembly from rna-seq data without a reference genome. Nat Biotechnol. 2011;29(7):644.

19. Gao CX, Yang BX, Zhang DD, Chen M, Tian JK. Enhanced metabolic process to indole alkaloids in Clematis terniflora DC after exposure to high level of UV-B irradiation followed by the dark. BMC Plant Biol. 2016;16:231.

20. Amirkhanian $V$, Tsai E. Use of a pen-shaped capillary gel electrophoresis cartridge for cost-effective DNA fragment analysis. Am Lab. 2012;44(7):16-7.

21. Yeh FC, Boyle TJB. Population genetic analysis of co-dominant and dominant markers and quantitative traits. Belg J Bot. 1997;129:157.

22. Liu K, Muse SV. Power marker: an integrated analysis environment for genetic marker analysis. Bioinformatics. 2005;21(9):2128-9.

23. Nei M, Li WH. Mathematical model for studying genetic variation in terms of restriction endonucleases. Proc Natl Acad Sci U S A. 1979;76(10):5269-73.

24. Wang W, Lu AM, Ren Y, Endress ME, Chen ZD. Phylogeny and classification of Ranunculales: evidence from four molecular loci and morphological data. Perspect Plant Ecol. 2009;11(2):81-110.

25. Wang W, Xie L. A revision of Clematis sect. Tubulosae (Ranunculaceae). Acta Phytotaxon Sin. 2007:45(4):425-57.

26. Sorkheh K, Dehkordi MK, Ercisli S, Hegedus A, Halász J. Comparison of traditional and new generation DNA markers declares high genetic diversity and differentiated population structure of wild almond species. Sci Rep. 2017;7(1):1-17

27. Jiang M, Zhou YQ, Li RR. ITS sequence analysis of eight medicinal plants in Clematis L. Chin Tradit Herbal Drugs. 2011;42(9):1802-6.

28. Wang L, Du HY, Li TZ, Wuyun TN. De novo transcriptome sequencing and identification of genes related to salt stress in Eucommia ulmoides Oliver. Trees. 2017:32(1):151-63.

29. Souframanien J, Reddy KS. De novo assembly, characterization of immature seed transcriptome and development of genic-SSR markers in black gram [Vigna mungo (L.) Hepper]. Plos One. 2015;10(6):e0128748.

30. Dong S, Liu Y, Niu J, Ning Y, Lin S, Zhang Z. De novo transcriptome analysis of the Siberian apricot (Prunus sibirica L.) and search for potential SSR markers by 454 pyrosequencing. Gene. 2014;544(2):220.

31. Zhang LS, Yang XN, Qi XN, Guo CH, Jing ZB. Characterizing the transcriptome and microsatellite markers for almond (Amygdalus communis L.) using the illumina sequencing platform. Hereditas. 2018;155(1):14.

32. Wu T, Luo S, Wang R, Zhong $Y, X u X$, Lin $Y, H e ~ X$, et al. The first illuminabased de novo transcriptome sequencing and analysis of pumpkin (Cucurbita moschata Duch.) and SSR marker development. Mol Breed. 2014; 34(3):1437-47.

33. Wang J, Chen Z, Jin S, Hu Z, Huang Y, Diao Y. Development and characterization of simple sequence repeat (SSR) markers based on a full-length cDNA library of napier grass (Pennisetum purpureum Schum). Genes \& Genom. 2017;3:1-9.

34. Yue X, Liu G, Zong Y, Teng Y, Cai D. Development of genic SSR markers from transcriptome sequencing of pear buds. J Zhejiang Univ Sci B. 2014; 5(4):303-12
35. Yang $Y$, Jing ZB, Ruan XF, Cheng JM. Development of simple sequence repeat markers in persimmon (Diospyros $\mathrm{L}$ ) and their potential use in related species. Genet Mol Res. 2015;14(1):609.

36. Miikeda O, Kita K, Handa T, Yukawa T. Phylogenetic relationships of Clematis (Ranunculaceae) based on chloroplast and nuclear DNA sequences. Bot J Linn Soc. 2006;152(2):153-68.

37. Li Y, Xu C, Lin X, Cui B, Wu R, Pang X. De novo assembly and characterization of the fruit transcriptome of Chinese jujube (Ziziphus jujuba Mill.) using 454 pyrosequencing and the development of novel tri-nucleotide SSR markers. Plos One. 2014;9(9):e106438.

38. Huang LL, Yang X, Sun P, Wen T, Hu SQ. The first illumina-based De novo transcriptome sequencing and analysis of safflower flowers. PLoS One. 2012; 7(6):e38653.

39. Chen H, Liu L, Wang L, Wang S, Somta P, Cheng X. Development and validation of EST-SSR markers from the transcriptome of adzuki bean (Vigna angularis). PLoS One. 2015;10(7):e0131939.

40. Li D, Zhi D, Bi Q, Liu X, Men Z. De novo assembly and characterization of bark transcriptome using illumina sequencing and development of EST-SSR markers in rubber tree (Hevea brasiliensis Muell. Arg.). Bmc Genomics. 2012; 13(1):192

Ready to submit your research? Choose BMC and benefit from:

- fast, convenient online submission

- thorough peer review by experienced researchers in your field

- rapid publication on acceptance

- support for research data, including large and complex data types

- gold Open Access which fosters wider collaboration and increased citations

- maximum visibility for your research: over $100 \mathrm{M}$ website views per year

At BMC, research is always in progress.

Learn more biomedcentral.com/submissions 